Research Article

\title{
Code Design for Moving Target-Detecting Radar in Nonhomogeneous Signal-Dependent Clutter
}

\author{
Jindong Zhang $\mathbb{D}$, Yin Du, and He Yan \\ College of Electronic and Information Engineering, Nanjing University of Aeronautics and Astronautics, Nanjing, \\ Jiangsu 210016, China \\ Correspondence should be addressed to Jindong Zhang; zjdjs@126.com
}

Received 3 March 2020; Revised 27 May 2020; Accepted 16 June 2020; Published 9 July 2020

Academic Editor: Kishin Sadarangani

Copyright $(92020$ Jindong Zhang et al. This is an open access article distributed under the Creative Commons Attribution License, which permits unrestricted use, distribution, and reproduction in any medium, provided the original work is properly cited.

In this paper, we investigate the code design problem of improving the detection performance of a moving target in the presence of nonhomogeneous signal-dependent clutter for moving target-detecting (MTD) radar systems. The optimization metric is constructed based on the signal to clutter and noise ratio (SCNR) of interpulse matched filtering. Under the frameworks of cyclic and majorization-minimization algorithms, we propose a novel algorithm, named CMMCODE, to tackle the code design optimization problem in the case of unknown precise target Doppler information and nonhomogeneous clutter. In the whitenoise case, the simplified algorithm is also given based on CMMCODE algorithm. The presented algorithm is computationally efficient and convergent. Numerical examples show the effectiveness of the proposed algorithms.

\section{Introduction}

Radars are required to deal with the simultaneous effects of receiver noise, signal-dependent clutter, and signal-independent interference, when detecting targets since echoes are received from the natural environment such as land, sea, and weather. The signal-dependent clutter can be seen as the convolution result of the transmitted signal and unwanted obstacles, whose scattering magnitude can be many orders larger than that of the target. Additionally, the signal-independent interference consists of various types of noise and jamming signals. Moving target detecting (MTD) is a mode of operation of a radar to discriminate a moving target against clutter. By taking advantage of the fact that Doppler frequency shift of a moving target is different from that of clutter, MTD technology plays an important role in modern radar systems [1]. Recently, it has been demonstrated that, with clutter statistical information, signal-to-clutter and noise ratio (SCNR) and detection performance for MTD can be improved by optimizing transmit power for pulses in a coherent processing interval (CPI) [2].

The transmit waveform design has been paid much attention to in radar research area in the last decades. The optimal radar waveform is synthesized based on different performance objectives. In the early 1960s, the problem of designing radar signals and receivers that are optimum for detecting a point target masked by a background of clutter returns and thermal noise has been investigated. The problem of choosing an optimum signal has been discussed in [3]. For detecting a particular target in the presence of additive signal-dependent clutter, the waveform optimization method, developed by Grieve and Guerci $[4,5]$, is evaluated in terms of the SCNR metric under a particular model of the system, clutter, and targets. The numerical solution of the optimal waveform for the T-72 and M1 main battle tanks detection was investigated in [6]. The idea of clutter suppression based on the waveform design has been extended to other radar systems. In [7], waveform design for a multiple-input multiple-output (MIMO) radar has also been studied, and this work considered the signal-dependent clutter and designed the optimal waveform based on minimum mean squared-error (MMSE) in two scenarios. In [8-12], waveform design and waveform scheduling problems in space-time adaptive processing (STAP) for airborne radar are formulated with a cost function, and least-squared solutions for the designed waveform were obtained. It 
should be pointed out that these works have discussed the waveform design problem for clutter suppression from the viewpoint of interpulse coding.

A lot of work has also been carried out to radar code design by intrapulse coding [13-20]. Reference [13] has dealt with the design of coded waveforms which optimize radar performances in the presence of colored Gaussian disturbance. Reference [14] considered the problem of a knowledge-aided (possibly cognitive) transmit signal and receive filter design for point-like targets in signal-dependent clutter. Reference [15] considered the worst case code design problem for clutter-free cases under a similarity constraint to a given code. The ideas of [15] were generalized in [16] where the PAR constraint was included. For designing code for signal-dependent clutter suppression for MTD in pulsedDoppler radar, Aubry et al., Karbasi et al., Aubry et al., and Cheng et al. [17-20] proposed to resort to average and worst case performance metrics and several algorithms to solve highly nonconvex design problems. In [21, 22], block successive minimization methods and maximum block improvement $(\mathrm{MBI})$ and of sequential optimization have also been considered in waveform design algorithm both in signal-dependent clutter for the MIMO system and for a spectrally crowded environment. In [23], the joint design of transmitting sequence(s) and receiving filters subject to the Peak-to-Average Ratio (PAR) constraint in radar and communications applications was conducted, and two algorithms based on the majorization-minimization (MM) method are proposed. The joint design of both transmitted waveforms and receive filters for a colocated multiple-inputmultiple-output (MIMO) radar with the existence of signaldependent interference and white noise is studied in [24]. Two algorithms based on the general MM method are developed to tackle the weighted side lobe level minimization problem in [25]. The joint design of the Doppler robust transmit sequence and receive filter based on the signal-tonoise-plus-interference (SINR) of the filter output is investigated in [26]. The robust joint design of the radar transmitted waveform and receive filter bank in a background of range-unambiguous signal-dependent clutter based on SINR is studied in [27].

The clutter model in these researches can be classified into two kinds, i.e., homogeneous and nonhomogeneous clutter, according to the properties of the clutter covariance matrix. Homogeneous clutter has an identical clutter covariance matrix for all resolution cells, and code design for homogeneous clutter can improve the detection performance for all cells. However, the clutter covariance matrix of one resolution cell in nonhomogeneous clutter may be different from that of the other cells. Therefore, code design for a certain range cell may deteriorate the others in nonhomogeneous clutter. In this paper, we will investigate the code design problem of detecting a moving target in the presence of nonhomogeneous clutter for MTD radar systems. Two different scenarios including general signal-independent interference and white noise are considered with the problem of detecting a moving target without precise Doppler frequency shift information. The optimization problems in this work are highly nonconvex and, therefore, firstly, made convex via relaxation of the original problems. Under the frameworks of cyclic algorithms [28-32] and MM algorithms [23, 25, 26, 33, 34], new algorithms are proposed to solve the convex problem. The work of this paper is summarized as follows:

(1) The effect of nonhomogeneous clutter and a moving target is considered. To our knowledge, code design for improving detection performance in nonhomogeneous clutter has not been addressed in the literature.

(2) The optimization metrics are constructed based on interpulse matched filtering, which is computationally efficient for coherent processing in a CPI. The corresponding optimization metric is given, and the connections between the SCNR and optimization metric are analytically addressed.

(3) Under the frameworks of majorization-minimization and cyclic algorithms, we propose new algorithms to tackle the code design optimization problem in the case of unknown precise target Doppler information and nonhomogeneous clutter. The presented algorithm is computationally efficient and convergent.

The rest of this paper is organized as follows. In Section 2, we derive the signal model and SCNR metric for MTD radar systems in a nonhomogeneous clutter scene. Code design for nonhomogeneous signal-dependent clutter is discussed in Section 3. This section also includes the problem formulation, problem transformation, lemmas, optimization method, and convergence analysis. Section 4 considers the white-noise case. Several numerical examples are provided in Section 5. Section 6 concludes this paper.

\section{Signal Model}

We assume that a pulsed-Doppler radar transmits a coherent burst of $N$ slow-time pulses. The $n$th transmitted baseband signal can be formulated as

$$
s\left(\widehat{t}, t_{n}\right)=a_{n} \operatorname{rect}\left(\frac{\widehat{t}-n T_{r}}{T_{p}}\right) \phi\left(\widehat{t}-n T_{r}\right),
$$

where $\widehat{t}$ and $t_{n}=n T_{r}$ denote the fast time and slow time, $0 \leq n \leq N-1, T_{p}$ and $T_{r}$ are the pulse duration and pulse repetition interval (PRI), respectively $\left(T_{r} \gg T_{p}\right)$, and $\operatorname{rect}(\cdot)$ is the rectangular pulse shaping function, expressed as

$$
\operatorname{rect}(\hat{t})= \begin{cases}1, & 0 \leq \widehat{t}<1, \\ 0, & \text { else where, }\end{cases}
$$

where $\phi(\hat{t})$ is the complex envelop of the transmit signal and $\left\{a_{n}\right\}_{n=0}^{N-1}$ are the weights that control the pulse power at the transmitter and will be tried to be designed.

The radar scene can be split into different cells in range and velocity domains by a pulsed-Doppler radar at the same time. Range cells are obtained by transmitting a waveform with a certain bandwidth, whereas velocity cells are obtained by utilizing a burst of pulses that have a high Doppler 
frequency resolution. Suppose that there exists $K$ different range cells and a point-like moving target lies in the $k$ th range cell $(1 \leq k \leq K)$. The corresponding backscatter signal can be expressed as

$$
\begin{aligned}
r_{k}\left(\widehat{t}, t_{n}\right)= & \alpha_{k} a_{n} \operatorname{rect}\left(\frac{\hat{t}-\tau_{k}-n T_{r}}{T_{p}}\right) e^{j 2 \pi\left(f_{c}+v_{k}^{t}\right)\left(\widehat{t}-\tau_{k}\right)} \\
& \cdot \phi\left(\hat{t}-\tau_{k}-n T_{r}\right)+c_{k}(t)+w(t),
\end{aligned}
$$

where $\alpha_{k}$ is the amplitude of the target echo, $\tau_{k}$ indicates the time delay of the $k$ th range cell, $v_{k}^{t}$ indicates the Doppler frequency of the moving target, $f_{c}$ is the transmitter carrier frequency, $c_{k}(t)$ is the clutter signal of the $k$ th range cell, and $w(t)$ represents the signal-independent interferences. In this paper, clutter is assumed to be range-unambiguous.

We further assume that the clutter, distributed across the Doppler frequency domain, is composed of many individual scattering points, which are statistically independent. Consequently, $c_{k}\left(\widehat{t}, t_{n}\right)$ can be written as

$$
c_{k}\left(\widehat{t}, t_{n}\right)=a_{n} \sum_{l=1}^{N_{k}^{c}} \rho_{k, l} \phi\left(\widehat{t}-\tau_{k}-n T_{r}\right) e^{j 2 \pi\left(f_{c}+v_{k, l}^{c}\right)\left(\widehat{t}-\tau_{k}\right)},
$$

where $N_{k}^{c}$ is the number of individual scatters in the $k$ th range cell, $\rho_{k, l}$ is the amplitude of the $l$ th scattering clutter point, and $v_{k, l}^{c}$ is the corresponding Doppler frequency.

Note that, after downconverted and demodulated in the receiver, the received baseband signals are first compressed in the range domain to separate echo reflected by different range cells, and then, Doppler processing is performed to distinguish moving targets from stationary clutter. In radar engineering, the received signal is sampled at the time delays corresponding to all the range cells under test, whose interval is determined by the range resolution of the radar system. The discrete-time received signal $\mathbf{r}_{k}$ for the $k$ th range cell corresponding to time delay $\tau_{k}$ can be written as

$$
\mathbf{r}_{k}=\widetilde{\alpha}_{k} \mathbf{a} \odot \mathbf{p}_{k}+\mathbf{a} \odot \mathbf{c}_{k}+\mathbf{w}_{k},
$$

where $\widetilde{\alpha}_{k}=\alpha_{k} e^{-j 2 \pi f_{c} \tau_{k}}, \mathbf{a}=\left[a_{0}, a_{1}, \ldots, a_{N-1}\right]^{T}$ is the code vector to be designed, $\mathbf{p}_{k}=\left[1, e^{j 2 \pi v_{k}^{t} T_{r}}, \ldots, e^{j 2 \pi(N-1) v_{k}^{t} T_{r}}\right]^{T}$ is the Doppler frequency shift vector of the target, $[\cdot]^{T}$ denotes the transpose of a vector/matrix, $\boldsymbol{c}_{k}$ is the vector corresponding to the clutter component in the $k$ th range cell, the vector $\mathbf{w}_{k}$ represents the signal-independent noise or interferences, and $\odot$ denotes the Hardmard (element-wise) product. A detailed description of the formation of $\mathbf{c}_{k}$ and $\mathbf{w}_{k}$ can be found in [2].

According to (5), the moving target detection problem can be given as the following binary hypothesis test:

$$
\begin{cases}H_{0}: & \mathbf{r}_{k}^{(0)}=\mathbf{a} \odot \mathbf{c}_{k}+\mathbf{w}_{k}, \\ H_{1}: & \mathbf{r}_{k}^{(1)}=\widetilde{\alpha}_{k} \mathbf{a} \odot \mathbf{p}_{k}+\mathbf{a} \odot \mathbf{c}_{k}+\mathbf{w}_{k} .\end{cases}
$$

Suppose that the covariance matrices of $\mathbf{c}_{k}$ and $\mathbf{w}_{k}$ (denoted by $\mathbf{C}_{k}$ and $\mathbf{M}_{k}$ ) are a priori known by using geographical, meteorological, or prescan information [35] and the scattering amplitude of the moving target $\alpha_{k}$ is a zero-mean complex Gaussian random variable with variance
$\sigma_{T, k}^{2}$. The matched filter detector which compares the likelihood ratio associated with the abovementioned hypothesis test is $\mathbf{h}_{k}=\mathbf{a} \odot \mathbf{p}_{k}$. The performance of this detector in the $k$ th range cell depends on the following SCNR metric, i.e.,

$$
\begin{aligned}
\operatorname{SCNR}_{k} & =\frac{\left|\mathbf{h}_{k}^{H}\left(\widetilde{\alpha}_{k} \mathbf{a} \odot \mathbf{p}_{k}\right)\right|^{2}}{E\left[\mathbf{h}_{k}^{H}\left(\mathbf{a} \odot \mathbf{c}_{k}+\mathbf{w}_{k}\right)\right]^{2}} \\
& =\frac{\sigma_{T, k}^{2} e}{\left(\mathbf{a} \odot \mathbf{p}_{k}\right)^{H}\left(\mathbf{M}_{k}+\mathbf{A} \mathbf{C}_{k} \mathbf{A}^{H}\right)\left(\mathbf{a} \odot \mathbf{p}_{k}\right)} \\
& =\frac{\sigma_{T, k}^{2} e}{\operatorname{tr}\left\{\mathbf{A}^{H}\left(\mathbf{M}_{k}+\mathbf{A} \mathbf{C}_{k} \mathbf{A}^{H}\right) \mathbf{A} \mathbf{p}_{k} \mathbf{p}_{k}^{H}\right\}}
\end{aligned}
$$

where $e$ denotes the maximum transmit energy, $\mathbf{A}=\operatorname{Diag}(\mathbf{a}), \operatorname{Diag}(\cdot)$ denotes the diagonal matrix formed by the entries of the vector argument, $[\cdot]^{H}$ denotes conjugate transpose of a vector/matrix, and $\operatorname{tr}(\cdot)$ denotes the trace of a matrix. Note that $\mathbf{A}=\operatorname{Diag}(\mathbf{a})$, and $e=\operatorname{tr}\left(\mathbf{A} \mathbf{A}^{H}\right)=\mathbf{a}^{H} \mathbf{a}$ is an energy constraint.

\section{Code Design for Nonhomogeneous Signal- Dependent Clutter}

3.1. Problem Formulation. Suppose that the Doppler frequency shift of the target $v_{k}^{t}$ is known and the variance difference of the scattering amplitude $\alpha_{k}$ is ignored; code design for improving the detection performance of the MTD radar system can be achieved by minimizing the following performance metric, which can be expressed as

$$
\left(\mathbf{a} \odot \mathbf{p}_{k}\right)^{H}\left(\mathbf{M}_{k}+\mathbf{A} \mathbf{C}_{k} \mathbf{A}^{H}\right)\left(\mathbf{a} \odot \mathbf{p}_{k}\right) .
$$

If the target Doppler frequency shift is unknown, but the considered interval $\Omega=\left[v_{k}^{t, \text { lower }}, v_{k}^{t \text {,upper }}\right]\left(v_{k}^{t, \text { lower }}\right.$ and $v_{k}^{t, \text { upper }}$ are upper and lower bounds, respectively) for the target Doppler frequency shift is known, the following design metric (referred to as average metric) can be used for such a case:

$$
\int_{\Omega}\left(\mathbf{a} \odot \mathbf{p}_{k}\right)^{H}\left(\mathbf{M}_{k}+\mathbf{A} \mathbf{C}_{k} \mathbf{A}^{H}\right)\left(\mathbf{a} \odot \mathbf{p}_{k}\right) f\left(v_{k}^{t}\right) d v_{k}^{t},
$$

where $f\left(v_{k}^{t}\right)$ is the probability density function (pdf) of $v_{k}^{t}$. It should be pointed out the mentioned interval $\Omega$ is a prior known based on prior known knowledge of the moving target type or prescan estimates of the target Doppler frequency shift. In common cases, $v_{k}^{t}$ is considered to be uniformly distributed over $\Omega$ to model the pdf of the target Doppler frequency shift.

Suppose that the target Doppler frequency shift is uniformly distributed over the interval $\Omega$ which is previously known; then, the discrete form of the design metric can also be given by

$$
\mathscr{M}_{k}=\frac{1}{L} \sum_{l=1}^{L}\left(\mathbf{a} \odot \mathbf{p}_{k}\left(v_{k, l}^{t}\right)\right)^{H}\left(\mathbf{M}_{k}+\mathbf{A} \mathbf{C}_{k} \mathbf{A}^{H}\right)\left(\mathbf{a} \odot \mathbf{p}_{k}\left(v_{k, l}^{t}\right)\right),
$$

where $L$ is the number of discrete Doppler frequency shift, $v_{k, l}^{t}=v_{k, l}^{t, \text { lower }}+(l-1) /(L-1)\left(v_{k, l}^{t \text {,upper }}-v_{k, l}^{t, \text { lower }}\right)$, $l=1,2, \ldots, L$. 
Until now, we have only considered the design metric for only one range cell under test, and for homogeneous clutter scene, the detection performance will be improved for all range cells. However, for the nonhomogeneous clutter scene, the covariance matrices $\left\{\mathbf{C}_{k}\right\}$ are different from each other. Code design for a certain range cell may result in a decreased SCNR and detection performance in other range cells. In cases with a nonhomogeneous clutter scene and a prior known Doppler frequency shift interval, the design metric can be represented as

$$
\sum_{k=1}^{K} \mathscr{M}_{k}\left(\mathbf{M}_{k}, \mathbf{C}_{k}\right)
$$

where $K$ is the number of all range cells under test.

Consider that the signal-independent noise or interferences usually have the same statistical characteristic, which implies that $\mathbf{M}_{1}=\mathbf{M}_{2}=\cdots=\mathbf{M}_{K}=\mathbf{M}_{0}$. With a drop the in the constant value for simplification, the abovementioned design metric can also be expressed as

$$
\sum_{k=1}^{K} \sum_{l=1}^{L}\left(\mathbf{a} \odot \mathbf{p}_{k}\left(v_{k, l}^{t}\right)\right)^{H}\left(\mathbf{M}_{0}+\mathbf{A} \mathbf{C}_{k} \mathbf{A}^{H}\right)\left(\mathbf{a} \odot \mathbf{p}_{k}\left(v_{k, l}^{t}\right)\right) .
$$

In a nonhomogeneous clutter scene, the SCNR expression is different from each other, and code design for a certain range cell may not optimize the SCNR in other range cells. Therefore, we should use the abovementioned design metric, which is the sum of clutter and signal-independent noise output of matched filters of all range cells, to evaluate the optimization performance.

The abovementioned design metric can also be transformed into the following expression, which is expressed as

$$
\mathbf{a}^{H} \mathbf{M a}+\mathbf{a}^{H} \mathbf{A C A}^{H} \mathbf{a},
$$

where

$$
\begin{aligned}
& \mathbf{M}=\sum_{k=1}^{K} \sum_{l=1}^{L} \mathbf{M}_{0} \odot\left(\mathbf{p}_{k}\left(v_{k, l}^{t}\right) \mathbf{p}_{k}\left(v_{k, l}^{t}\right)^{H}\right), \\
& \mathbf{C}=\sum_{k=1}^{K} \sum_{l=1}^{L} \mathbf{C}_{k} \odot\left(\mathbf{p}_{k}\left(v_{k, l}^{t}\right) \mathbf{p}_{k}\left(v_{k, l}^{t}\right)^{H}\right) .
\end{aligned}
$$

To optimize the detection performance in a nonhomogeneous clutter scene, the optimization problem can be formulated under an energy constraint:

$$
\begin{aligned}
\min _{\mathbf{a}} & \mathbf{a}^{H} \mathbf{M a}+\mathbf{a}^{H} \mathbf{A C A} \mathbf{A}^{H} \mathbf{a} \\
\text { s.t. } & \mathbf{a}^{H} \mathbf{a}=e .
\end{aligned}
$$

The objective function of the abovementioned optimization problem consists of a quadratic form $\mathbf{a}^{H} \mathbf{M a}$ and a quartic form $\mathbf{a}^{H} \mathbf{A} \mathbf{C A} \mathbf{A}^{H}$ a. Because of the quartic form in an objective function, it is difficult to optimize this problem directly. As the objective function is highly nonconvex, this problem is difficult to tackle. In this paper, we try to transform this nonconvex problem by using some important inequalities.

\subsection{Lemmas for Optimization}

Lemma 1. Let $\mathbf{R}$ be an $N \times N$ Hermitian and positive definite matrix; then, for any point $\mathbf{x}, \mathbf{x}_{0} \in \mathbb{C}^{N}$, the quadratic form $\mathbf{x}^{H} \mathbf{R} \mathbf{x}$ is majorized by

$$
\sigma_{1} \mathbf{x}^{H} \mathbf{I} \mathbf{x}+2 \operatorname{Re}\left(\mathbf{x}^{H}\left(\mathbf{R}-\sigma_{1} \mathbf{I}\right) \mathbf{x}_{0}\right)+\mathbf{x}_{0}^{H}\left(\sigma_{1} \mathbf{I}-\mathbf{R}\right) \mathbf{x}_{0},
$$

where $\sigma_{1}$ is the largest eigenvalue of $\mathbf{R}$. Proof of Lemma 1 is presented in Appendix A.

Lemma 2. Let $\mathbf{Q}$ be an $N \times N$ Hermitian and positive definite matrix and $\mathbf{X}_{1}=\operatorname{Diag}\left(\mathbf{x}_{1}\right)$ and $\mathbf{X}_{0}=\operatorname{Diag}\left(\mathbf{x}_{0}\right)$ be diagonal matrices, $\mathbf{x}_{1}, \mathbf{x}_{0} \in \mathbb{C}^{N}$; then, for any point $\mathbf{x} \in \mathbb{C}^{N}$, we have

$$
\left|\mathbf{x}^{H} \mathbf{X}_{0} \mathbf{Q} \mathbf{X}_{0}^{H} \mathbf{x}_{1}\right|^{2} \leq\left(\mathbf{x}^{H} \mathbf{X}_{0} \mathbf{Q} \mathbf{X}_{0}^{H} \mathbf{x}\right) \cdot\left(\mathbf{x}_{1}^{H} \mathbf{X}_{0} \mathbf{Q} \mathbf{X}_{0}^{H} \mathbf{x}_{1}\right),
$$

and the equality is obtained if $\mathbf{x}=\mathbf{x}_{1}$.

Lemma 2 can be directly concluded via Cauchy-Shwarz inequality.

Theorem 1. Let $\mathbf{Q}$ be an $N \times N$ Hermitian and positive definite matrix and $\mathbf{X}=\operatorname{Diag}(\mathbf{x})$ be a diagonal matrix $\mathbf{x}_{0} \in \mathbb{C}^{N}$; then, the form $\mathbf{x}^{H} \mathbf{X} \mathbf{Q} \mathbf{X}^{H} \mathbf{x}$ is majorized by

$$
2 \operatorname{Re}\left[\mathbf{x}^{H} \mathbf{X}_{0} \mathbf{Q}^{*} \mathbf{X}_{0}^{H} \mathbf{x}_{0}\right]-4 \xi_{1} \operatorname{Re}\left(\mathbf{x}^{H} \mathbf{x}_{0}\right)+\kappa\left(\mathbf{x}_{0}\right)
$$

where $\kappa\left(\mathbf{x}_{0}\right)=4 \xi_{1} e-\mathbf{x}_{0}^{H} \mathbf{X}_{0} \mathbf{Q}^{*} \mathbf{X}_{0}^{H} \mathbf{x}_{0}, e=\|\mathbf{x}\|^{2}=\left\|\mathbf{x}_{0}\right\|^{2}$, and $\xi_{1}$ is the largest eigenvalue of $\mathbf{X} \mathbf{Q} \mathbf{X}^{H}$. Proof of Theorem 1 is presented in Appendix $B$.

Lemma 3. Let $\mathbf{Q}$ be $N \times N$ Hermitian and positive definite matrices, $\mathbf{X}=\operatorname{Diag}(\mathbf{x})$ is a diagonal matrix, and $e=\|\mathbf{x}\|^{2}$; then, the matrix $\mathbf{X Q X} \mathbf{X}^{H}$ is still a positive definite matrix. The largest eigenvalues $\xi_{1}$ of $\mathbf{X Q \mathbf { X } ^ { H }}$ satisfy

$$
\lambda_{\max }\left(\mathbf{X} \mathbf{Q} \mathbf{X}^{H}\right) \leq e \lambda_{\max }(\mathbf{Q}) \text {, }
$$

where $\lambda_{\max }(\cdot)$ denotes the largest eigenvalues of a matrix. In the proof of Lemma 3, the inequality follows by Weil's monotonicity theorem ("Matrix Analysis," p. 184, Theorem 4.3.7, applied with $j=k=n$ ) and then uses, directly, the bound on $\lambda_{\max }\left(\mathbf{X Q X} \mathbf{X}^{H}\right)[36,37]$.

\subsection{Majorization-Minimization-Based Optimization} Method. The majorization-minimization (MM) method can be used to solve optimization problems that are difficult to tackle directly.

Let $\mathbf{a}^{(k)}$ and $\mathbf{A}^{(k)}$ be at the $k$ th iteration. By applying Lemma 1 and Theorem 1, the objective function in (15) can be majorized by the following form:

$$
\begin{aligned}
\mu\left(\mathbf{a}, \mathbf{a}^{(k)}\right)= & 2 \operatorname{Re}\left[\mathbf{a}^{H}\left(\mathbf{M}-\alpha_{1} \mathbf{I}\right) \mathbf{a}^{(k)}\right]+2 \operatorname{Re}\left[\mathbf{a}^{H}\left(\mathbf{A}^{(k)} \mathbf{C}^{*} \mathbf{A}^{(k) H}-2 \beta_{1} \mathbf{I}\right) \mathbf{a}^{(k)}\right] \\
& +\left(2 \alpha_{1}+4 \beta_{1}\right) e-\mathbf{a}^{(k) H} \mathbf{M} \mathbf{a}^{(k)}-\mathbf{a}^{(k) H} \mathbf{A}^{(k)} \mathbf{C}^{*} \mathbf{A}^{(k) H} \mathbf{a}^{(k)},
\end{aligned}
$$


where $\alpha_{1}$ is the largest eigenvalue of $\mathbf{M}$ and $\beta_{1}$ is the largest eigenvalue of $\mathbf{A} \mathbf{C}^{*} \mathbf{A}^{H}$. According to Lemma 5, we have $\beta_{1} \leq e \eta_{1}$, and $\eta_{1}$ is the largest eigenvalue of $\mathbf{C}$.

By ignoring the constant items in (20), the majorized optimization problem of (15) can be given by

$$
\begin{array}{ll}
\min _{\mathbf{a}} & \operatorname{Re}\left[\mathbf{a}^{H}\left(\mathbf{M}-\alpha_{1} \mathbf{I}\right) \mathbf{a}^{(k)}\right]+\operatorname{Re}\left[\mathbf{a}^{H}\left(\mathbf{A}^{(k)} \mathbf{C}^{*} \mathbf{A}^{(k) H}-2 \beta_{1} \mathbf{I}\right) \mathbf{a}^{(k)}\right] \\
\text { s.t. } & \mathbf{a}^{H} \mathbf{a}=e,
\end{array}
$$

which can be rewritten as

$$
\begin{array}{ll}
\min _{\mathbf{a}} & \operatorname{Re}\left[\mathbf{a}^{H}\left(\mathbf{M}+\mathbf{A}^{(k)} \mathbf{C}^{*} \mathbf{A}^{(k) H}-\left(\alpha_{1}+2 \beta_{1}\right) \mathbf{I}\right) \mathbf{a}^{(k)}\right] \\
\text { s.t. } & \mathbf{a}^{H} \mathbf{a}=e .
\end{array}
$$

This minimization problem can be transformed into a simple form:

$$
\begin{aligned}
\min _{\mathbf{a}} & \left\|\mathbf{a}^{(k+1)}-\mathbf{b}^{(k)}\right\|_{2} \\
\text { s.t. } & \mathbf{a}^{H} \mathbf{a}=e,
\end{aligned}
$$

where

$$
\mathbf{b}^{(k)}=-\left(\mathbf{M}+1 \mathbf{A}^{(k)} \mathbf{C}^{*} \mathbf{A}^{(k) H}-\left(\alpha_{1}+2 \beta_{1}\right) \mathbf{I}\right) \mathbf{a}^{(k)} .
$$

The closed-form ${ }_{2}$ solution of (23) is given by $\mathbf{a}^{(k+1)}=\sqrt{e} \mathbf{b}^{(k)} /\left\|\mathbf{b}^{(k)}\right\|_{2}^{2}$.

Now, we summarize the algorithm, and the corresponding steps are given in Table 1 .

In Table $1, \mathbf{a}^{(0)}$ is the initialized code, $\alpha_{1}$ is the largest eigenvalue of $\mathbf{M}, \beta_{1}$ is the largest eigenvalue of $\mathbf{A C}^{*} \mathbf{A}^{H}, \mathbf{b}^{(k)}$ is the calculated code of the $k$ th iteration, and $\mathbf{a}^{(k+1)}$ is the optimized code of the $k$ th iteration.

3.4. Convergence Analysis. According to the majorizationminimization scheme, the sequence of the objective function values $f\left(\mathbf{a}^{(k)}\right)$ at $\mathbf{a}^{(k)}$ is nonincreasing, and $f\left(\mathbf{a}^{(k)}\right)$ is guaranteed to converge to a finite value by

$$
f\left(\mathbf{a}^{(k+1)}\right) \leq u\left(\mathbf{a}^{(k+1)}, \mathbf{a}^{(k)}\right) \leq u\left(\mathbf{a}^{(k)}, \mathbf{a}^{(k)}\right)=f\left(\mathbf{a}^{(k)}\right) .
$$

Lemma 4. The optimization problem in (15) is equivalent to

$$
\begin{aligned}
\min _{\mathbf{a}} & \tilde{f}(\mathbf{a})=\mathbf{a}^{H} \mathbf{M a}+\mathbf{a}^{H} \mathbf{A C A}^{H} \mathbf{a}+\left(2 \alpha_{1}+8 \beta_{1}\right) \mathbf{a}^{H} \mathbf{a} \\
\text { s.t. } & \mathbf{a}^{H} \mathbf{a}=e .
\end{aligned}
$$

Since the added item is just a constant, it will not affect the optimal solution of (26), and vice versa. By ignoring the constant item, (26) is majorized by

$$
\begin{aligned}
\widetilde{\mu}\left(\mathbf{a}, \mathbf{a}^{(k)}\right)= & 2 \operatorname{Re}\left[\mathbf{a}^{H}\left(\mathbf{M}-\alpha_{1} \mathbf{I}\right) \mathbf{a}^{(k)}\right]+4 \operatorname{Re}\left[\mathbf{a}^{H}\left(\mathbf{A}^{(k)} \mathbf{C}^{*} \mathbf{A}^{(k) H}-2 \beta_{1} \mathbf{I}\right) \mathbf{a}^{(k)}\right] \\
& -\mathbf{a}^{(k) H} \mathbf{M} \mathbf{a}^{(k)}-2 \mathbf{a}^{(k) H} \mathbf{A}^{(k)} \mathbf{C}^{*} \mathbf{A}^{(k) H} \mathbf{a}^{(k)}+\left(2 \alpha_{1}+8 \beta_{1}\right) \mathbf{a}^{(k) H} \mathbf{a}^{(k)} .
\end{aligned}
$$

Theorem 2. The limit point $\mathbf{a}^{(\infty)}$ of the convergence sequence $\left\{\mathbf{a}^{(k)}\right\}$ is a stationary point of (15) and satisfies

$$
\nabla \tilde{f}\left(\mathbf{a}^{(\infty)}\right)^{T} \mathbf{a}^{(\infty)} \geq 0,
$$

where $\nabla(\cdot)$ denotes the first-order derivation [22].

Proof of Theorem 2 is presented in Appendix C.

\section{Code Design for the White-Noise Case}

A special case for code design in a MTD radar system is that the signal-dependent interference only contains receiver noise, which is white and statistically independent with each other. In this case, let $\mathbf{M}=\rho_{n}^{2} \mathbf{I}$, where $\rho_{n}^{2}$ is the noise power. Note that $\mathbf{a}^{H} \mathbf{M a}=\rho_{n}^{2} e$, and accordingly, the optimization problem in (15) is simplified into

$$
\begin{array}{ll}
\underset{\mathbf{s}}{\min } & \mathbf{s}^{T} \mathbf{C s} \\
\text { s.t. } & \sum_{n=1}^{N} s_{n}=e, \quad s_{n} \geq 0,
\end{array}
$$

where $\mathbf{s}=\left[s_{0}, s_{1}, \ldots, s_{N-1}\right]^{T}$ and $s_{n}=\left|a_{n}\right|^{2}$. It is easy to observe that the objective function in (29) is invariant to a phase-shift version of the code vector a. This means only amplitude modulation in slow-time pulses affects the corresponding optimization metric.

We can obtain a lemma similar to Lemma 2, which is described as follows.

Lemma 5. Let $\mathbf{R}$ be an $N \times N$ Hermitian and positive definite matrix; then, for any point $\mathbf{x}, \mathbf{x}_{0} \in \mathbb{R}^{N}$, the quadratic form $\mathbf{x}^{T} \mathbf{R} \mathbf{x}$ is majorized by

$$
\sigma_{1} \mathbf{x}^{T} \mathbf{I} \mathbf{x}+2 \operatorname{Re}\left(\mathbf{x}^{T}\left(\mathbf{R}-\sigma_{1} \mathbf{I}\right) \mathbf{x}_{0}\right)+\mathbf{x}_{0}^{T}\left(\sigma_{1} \mathbf{I}-\mathbf{R}\right) \mathbf{x}_{0},
$$

where $\sigma_{1}$ is the largest eigenvalue of $\mathbf{R}$.

By ignoring the constant item in (30), the majorized problem of (29) can be given by

$$
\begin{array}{ll}
\min _{\mathbf{s}} & \mathbf{s}^{T} \mathbf{s}+\operatorname{Re}\left[\mathbf{s}^{T}\left(\mathbf{C}-\alpha_{1} \mathbf{I}\right) \mathbf{s}^{(k)}\right] \\
\text { s.t. } & \sum_{n=1}^{N} s_{n}=e, \quad s_{n} \geq 0 .
\end{array}
$$

Let $2 \mathbf{d}^{(k)}=\operatorname{Re}\left[\left(\mathbf{C}-\alpha_{1} \mathbf{I}\right) \mathbf{s}^{(k)}\right]=\left[\operatorname{Re}(\mathbf{C})-\alpha_{1} \mathbf{I}\right] \mathbf{s}^{(k)}$, and the objective function of (31) is equivalent to

$$
\begin{aligned}
\mathbf{s}^{T} \mathbf{s}+\operatorname{Re}\left[\mathbf{s}^{T}\left(\mathbf{C}-\alpha_{1} \mathbf{I}\right) \mathbf{s}^{(k)}\right] & =\mathbf{s}^{T} \mathbf{s}+\mathbf{s}^{T}\left[\operatorname{Re}(\mathbf{C})-\alpha_{1} \mathbf{I}\right] \mathbf{s}^{(k)} \\
& =\sum_{n=1}^{N} s_{n}^{2}+2 s_{n} d_{n}^{(k)}=\sum_{n=1}^{N}\left(s_{n}+d_{n}^{(k)}\right)^{2}-\sum_{n=1}^{N} d_{n}^{(k) 2}=\left\|\mathbf{s}+\mathbf{d}^{(k)}\right\|_{2}^{2}-\left\|\mathbf{d}^{(k)}\right\|_{2}^{2},
\end{aligned}
$$


TABLE 1: The proposed algorithm for code design in nonhomogeneous clutter.

Step 1: set $k=0$, and initialize $\mathbf{a}^{(0)}$

Step 2: calculate $\alpha_{1}$ and $\beta_{1}$

Step 3: $\mathbf{b}^{(k)}=-\left(\mathbf{M}+\mathbf{A}^{(k)} \mathbf{C}^{*} \mathbf{A}^{(k) H}-\left(\alpha_{1}+2 \beta_{1}\right) \mathbf{I}\right) \mathbf{a}^{(k)}$

Step 4: $\mathbf{a}^{(k+1)}=\sqrt{e} \mathbf{b}^{(k)} /\left\|\mathbf{b}^{(k)}\right\|_{2}^{2}$

Step 5: $k=k+1$

Step 6: repeat steps 3-5 until convergence

where $\|\cdot\|_{2}$ denotes the $\ell_{2}$ norm.

Consequently, the optimization problem is also equivalent to

$$
\begin{array}{ll}
\min _{\mathbf{s}} & \left\|\mathbf{s}+\mathbf{d}^{(k)}\right\|_{2}^{2} \\
\text { s.t. } & \sum_{n=1}^{N} s_{n}=e, \quad s_{n} \geq 0 .
\end{array}
$$

The closed-form solution of (33) is given by $\mathbf{s}=-\mathbf{d}^{(k)}$. Considering the nonnegative restriction of $s_{n}$, the suboptimal solution of (33) can be written as

$$
\tilde{\mathbf{d}}_{n}^{(k+1)}= \begin{cases}-\mathbf{d}_{n}^{(k)}, & \mathbf{d}_{n}^{(k)} \leq 0, \\ 0, & \mathbf{d}_{n}^{(k)}>0 .\end{cases}
$$

Now, we summarize the algorithm, and the corresponding steps are given in Table 2.

In Table 2, $\mathbf{s}^{(0)}$ is the initialized code, $\alpha_{1}$ is the largest eigenvalue of $\mathbf{M}, \mathbf{f}^{(k)}$ is the calculated code of the $k$ th iteration, and $\mathbf{s}_{n}^{(k+1)}$ is the optimized code of the $k$ th iteration.

\section{Numerical Examples}

Numerical examples are provided to demonstrate the detection performance improvement of the proposed algorithms. Two code design examples including the general signal-independent interference and white noise are given. In the general signal-independent interference example, the entries of the corresponding covariance matrix $\mathbf{M}_{0}$ can be generated by a first-order autoregressive process with parameters $\rho_{\text {int }}=0.5$ and $p_{\text {int }}=1$, as well as a white noise at the receiver with the variance $\sigma_{n}^{2}$ :

$$
\mathbf{M}_{0}(m, n)=\sigma_{n}^{2} \delta[m-n]+p_{\text {int }} \rho_{\text {int }}^{|m-n|}, \quad 1 \leq m, n \leq N,
$$

where $\delta[m-n]$ is the discrete Dirac function, $\sigma_{n}^{2}=0.01$.

In the white-noise example, (35) is simplified into

$$
\mathbf{M}_{0}(m, n)=\sigma_{n}^{2} \delta[m-n], \quad 1 \leq m, n \leq N .
$$

To compare the performance improvement between the coded and uncoded cases, we define the transmit code as $\mathbf{a}_{0}=\sqrt{e / N} \mathbf{1}_{N \times 1}$, and the average metric for evaluation can be rewritten as

$$
\text { Average Metric }=\sum_{k=1}^{K} \mathscr{M}_{k}\left(M_{k}, C_{k}\right)
$$

This metric also indicates the algorithm performance in terms of merit (7).
TABLE 2: The proposed algorithm for code design in nonhomogeneous clutter and white noise.

Step 1: set $k=0$, and initialize $\mathbf{s}^{(0)}$

Step 2: calculate $\alpha_{1}$

Step 3: $\mathbf{d}^{(k)}=\operatorname{Re}\left[\left(\mathbf{C}-\alpha_{1} \mathbf{I}\right) \mathbf{s}^{(k)}\right] / 2$

Step 4: $\tilde{\mathbf{d}}_{n}^{(k+1)}= \begin{cases}-\mathbf{d}_{n}^{(k)}, & \mathbf{d}_{n}^{(k)} \leq 0, \\ 0, & \mathbf{d}_{n}^{(k)}>0\end{cases}$

Step 5: $\mathbf{s}_{n}^{(k+1)}=\tilde{\mathbf{d}}_{n}^{(k+1)} / \sum\left(\tilde{\mathbf{d}}_{n}^{(k+1)}\right)$

Step 5: $k=k+1$

Step 6: repeat steps 3-5 until convergence, $a_{n}=\sqrt{s_{n}}$

5.1. Homogeneous Clutter Case. Although CMMCODE algorithm is designed for the radar code for a nonhomogeneous clutter case, this algorithm can also be applied for obtaining optimal codes for the homogeneous clutter case. The goodness of the resultant codes is investigated by comparing with uncoded system, CoRe, and CADCODE algorithms in [2]. In this example, the entries of the $k$ th clutter covariance matrix $\mathbf{C}_{k}$ can be given by [38]:

$$
C_{k}(m, n)=\sigma_{c}^{2} \rho_{c}^{(k)|m-n|^{2}}, \quad 1 \leq m, n \leq N,
$$

where $\sigma_{c}^{2}=1$ and $\rho_{c}^{(k)}$ is a random number, representing the clutter characteristic [1]. For the moving target, we also assume that $v_{k}^{t}$ is uniformly distributed over $\Omega=\left[v^{t, \text { lower }}, v^{t \text {,upper }}\right]$, where $v^{t, \text { lower }}=-0.5$, and $v^{t \text {,upper }}=0.5$.

It is no surprise that the coded system, exploring the resultant codes of CoRe, CADCODE, and CMMCODE algorithms, outperform the uncoded system. Additionally, we note that average metric of the codes of CoRe, CADCODE, and CMMCODE are almost the same in Figure 1(a), with slightly negligible differences. This reveals that the convergence performance the CMMCODE algorithm is as good as CADCODE and CoRe algorithms. The saturation phenomenon in Figure 1(a) is also observed. This indicates that the increase in the average metric is negligible for sufficiently large values of the transmit energy $e$ (i.e., $e>25 \mathrm{~dB}$ ).

We also investigate the detection probability [39] of CoRe, CADCODE, CMMCODE algorithms, compared with that of the uncoded system. To this end, we consider the target with $\sigma_{T}^{2}=10$ and transmit energy $e=10$, and use 1000 sets of random generated data to simulate the receiver operating characteristic (ROC). ROCs of matched filter [40] with the resultant codes of CoRe, CADCODE, CMMCODE algorithms, and the uncoded code are plotted in Figure 1(b). As expected, the detection probabilities obtained by CoRe, CADCODE, CMMCODE algorithms outperform that of the uncoded system. Negligible differences can be observed between ROCs associated with various resultant codes.

In Figure 2, the convergence of CADCODE and CMMCODE is shown. We can find that the convergence speed of CMMCODE is faster than that of CADCODE. Considering that CMMCODE only involves complex multiplication and addition, the computational complexity of CMMCODE is lower than that of CADCODE.

5.2. Nonhomogeneous Clutter Case. In this example, we assume there exist 3 different range cells under the detection 


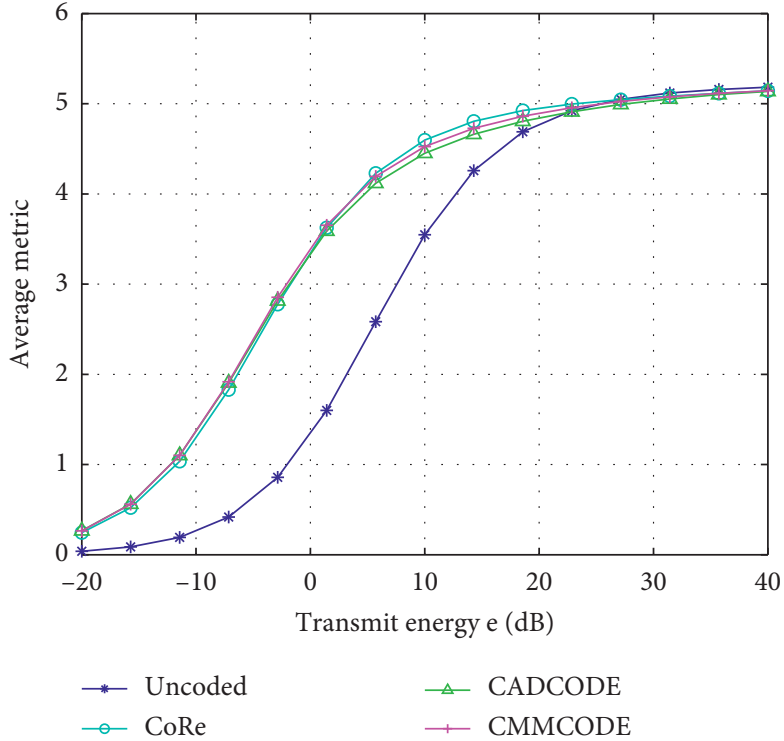

(a)

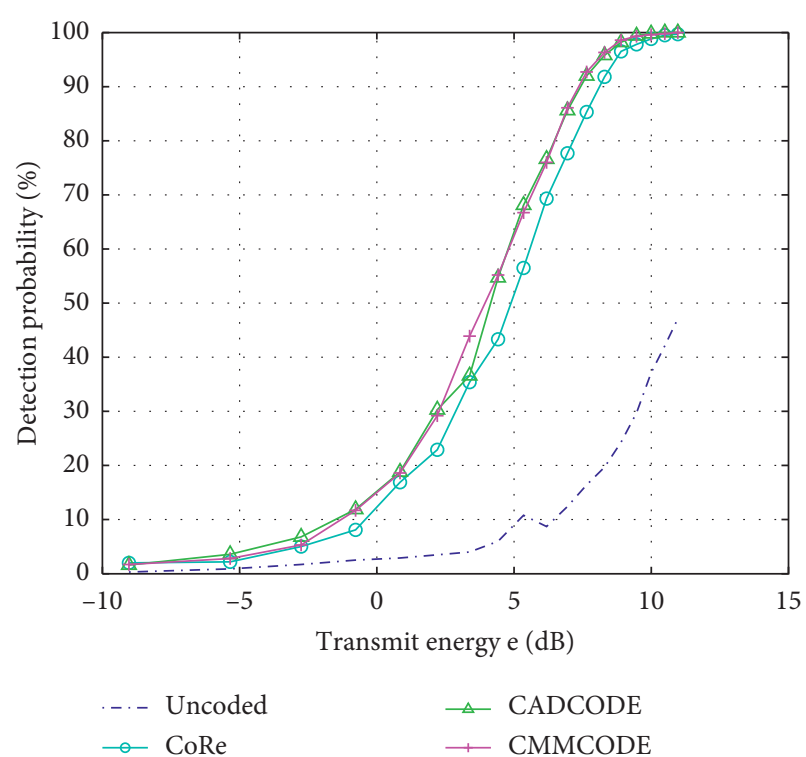

(b)

Figure 1: (a) Average metric vs. transmit energy $e$ for resultant codes of CoRe, CADCODE, and CMMCODE algorithms and the uncoded system; (b) detection probability for a matched filter detector.

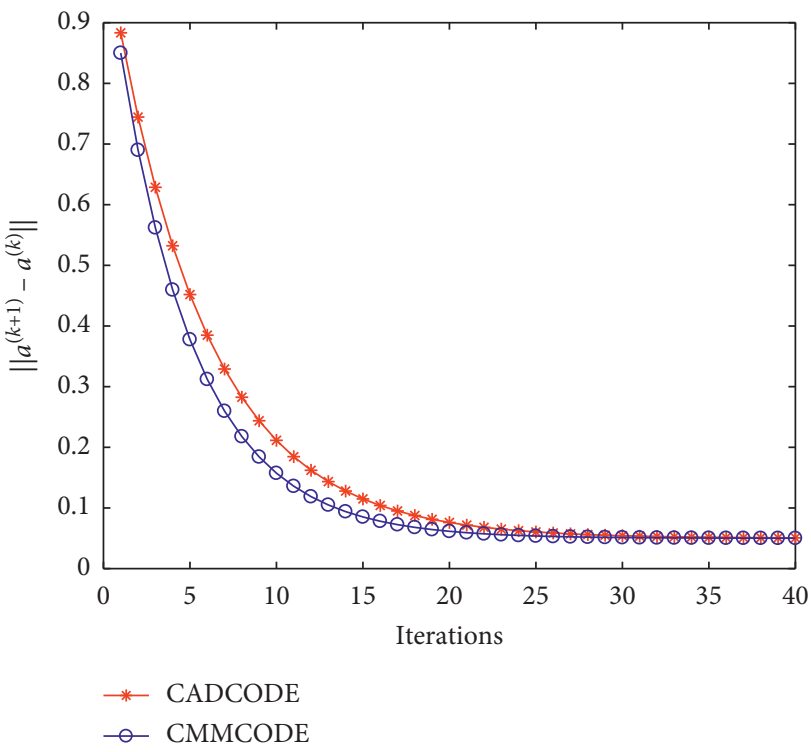

Figure 2: Convergence of CADCODE and CMMCODE.

test. To demonstrate the effectiveness of code design, the spectrum model of the clutter is assumed to be Gaussian, and its corresponding covariance coefficient is given by

$$
C_{k}(m, n)=e^{-2\left(\pi \sigma_{k}^{c}(m-n) T_{r}\right)^{2}} e^{j 2 \pi v_{k, 0}^{c}(m-n) T_{r}},
$$

where $\sigma_{k}^{c}$ denotes the spectrum width of the clutter of the $k$ th range cell and $v_{k, 0}^{c}$ indicates the center frequency of the clutter. We assume that there exist 3 different range cells, and $\sigma_{1}^{c}=40, \sigma_{2}^{c}=8, \sigma_{3}^{c}=15, v_{1,0}^{c}=-50 \mathrm{~Hz}, v_{2,0}^{c}=0 \mathrm{~Hz}$, and $v_{3,0}^{c}=25 \mathrm{~Hz}$. The pulse repetition interval $T_{r}$ equals to $0.01 \mathrm{~s}$. The interested target Doppler frequency lies in $[-10,10] \mathrm{Hz}$. The clutter spectrum shapes of 3 different range cells are plotted in Figure 3.

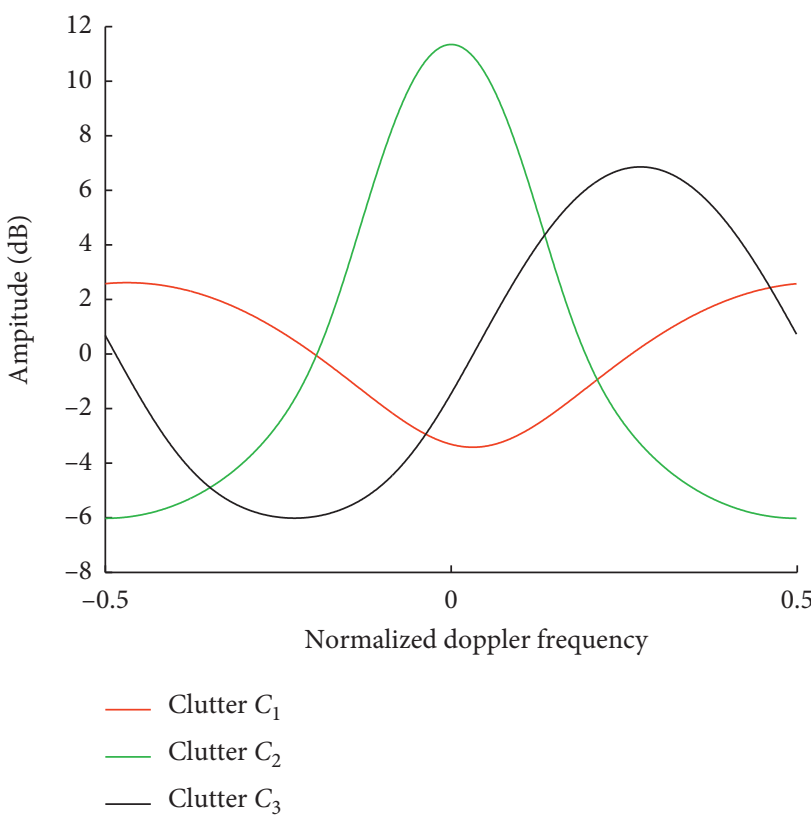

FIgURE 3: Clutter spectrum shape of different range cells.

Considering that CoRe and CADCODE algorithms can only synthesis code according to a certain clutter covariance matrix, we choose CADCODE algorithm for comparing the average metric and average detection probability with CMMCODE algorithm in a nonhomogeneous clutter case. Average detection probability (ADP) is defined to be the mean value of the detection probability in a different range cell and expressed as

$$
\mathrm{ADP}=\frac{1}{K} \sum_{k=1}^{K} P_{d}\left(\mathbf{M}_{k}, \mathbf{C}_{k}\right) \text {. }
$$

In Figures 4(a) and 4(b), we note that the resultant codes in CMMCODE algorithm own the highest average metric 


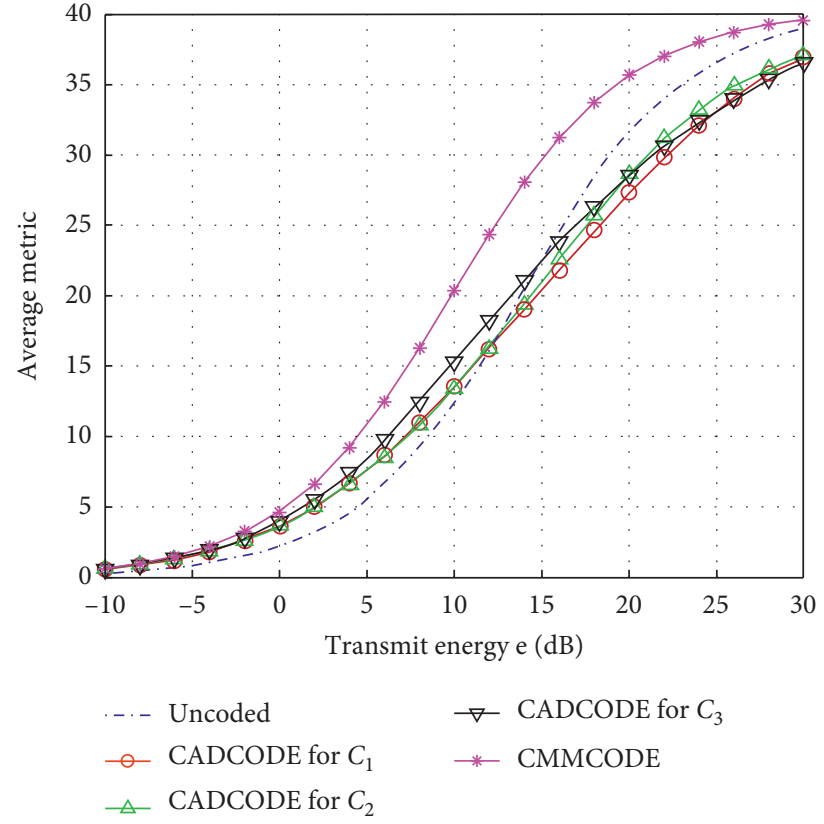

(a)

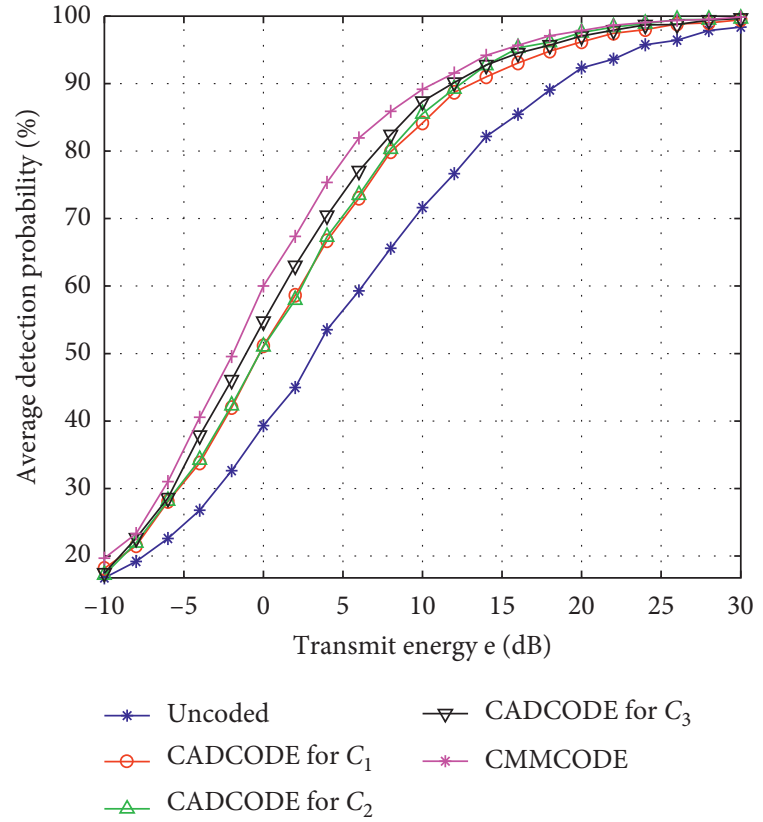

(b)

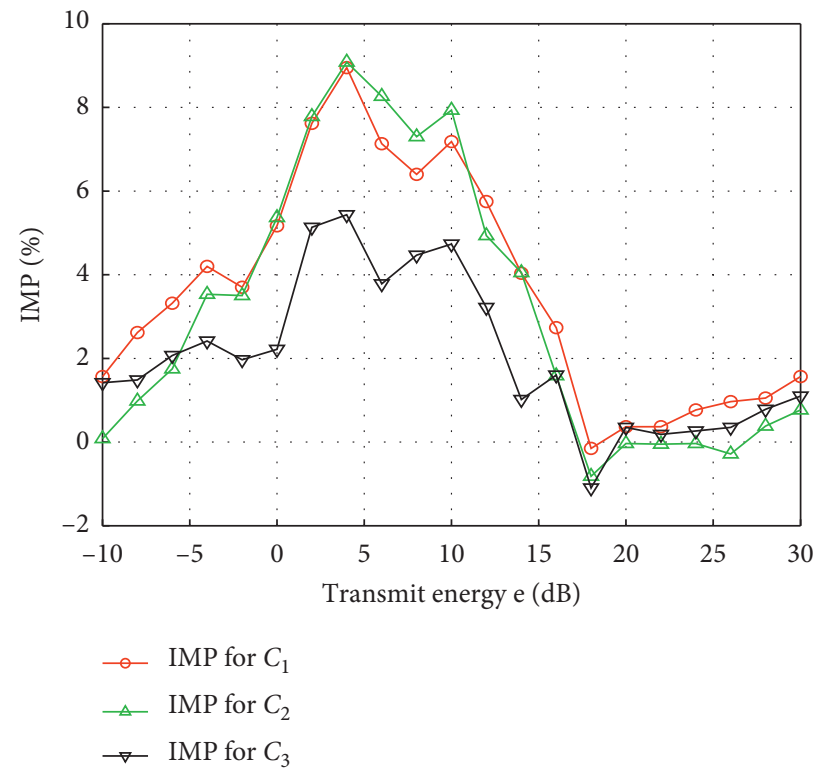

(c)

Figure 4: (a) Average metric of the resultant codes of CoRe, CADCODE, and CMMCODE algorithms and the uncoded system for 3 range cells; (b) average detection probability of an adaptive matched filter detector; and (c) IMP factor for different range cells.

and best detection performance, and the average metric of the resultant codes in CADCODE algorithm is slightly lower than the former. It can be easily seen that the code in CADCODE algorithm is designed according to the clutter covariance matrix of a preset range cell. However, the synthesized code in CADCODE algorithm can only have high metric in the corresponding range cell. Meanwhile, the codes in CMMCODE algorithm demonstrate adaptivity in all range cells.

To show the performance improvement of SCNR, the metric IMP is defined as
$\operatorname{IMP}\left(C_{k}\right)=$ Average Metric $(\mathrm{CMMCODE})-$ Average $\operatorname{Metri}\left(C_{k}\right)$.

Figure 4(c) demonstrates the IMP metric performance of the resultant codes of CMMCODE algorithm, compared with that of the CADCODE algorithm. It can be observed that the average detection probability of the resultant code of CMMCODE algorithm is 9 percent higher than that of the resultant codes of CADCODE algorithm in the maximum extent. It can also be concluded that this improvement is not available in all transmit energy ranges, and best 


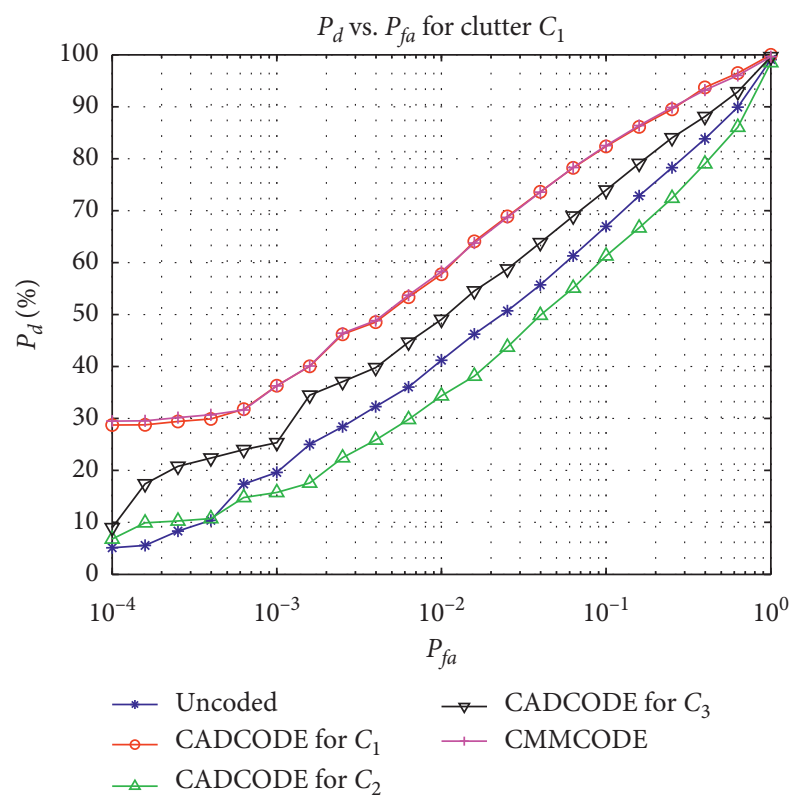

(a)

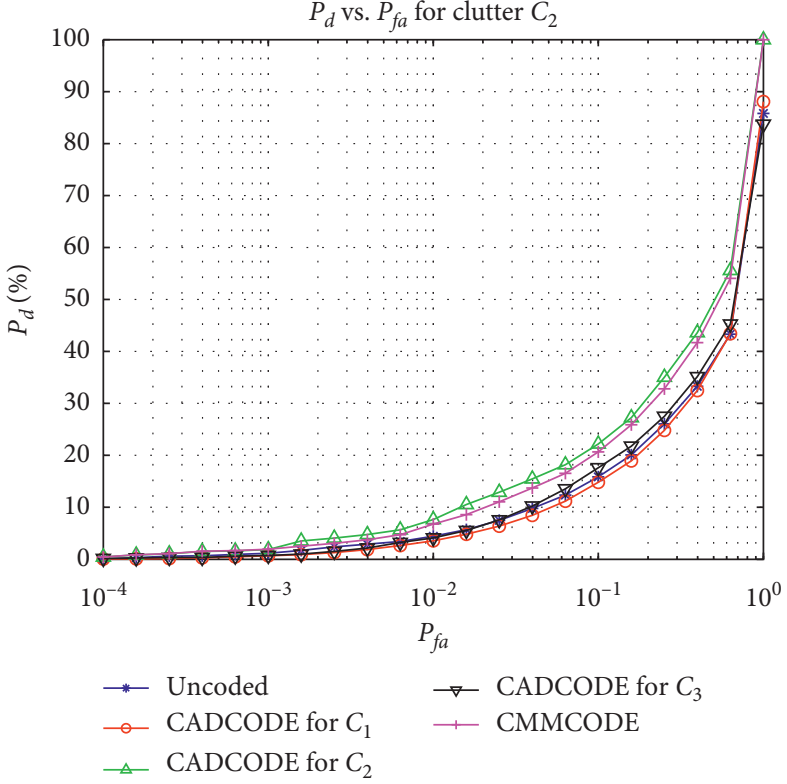

(b)

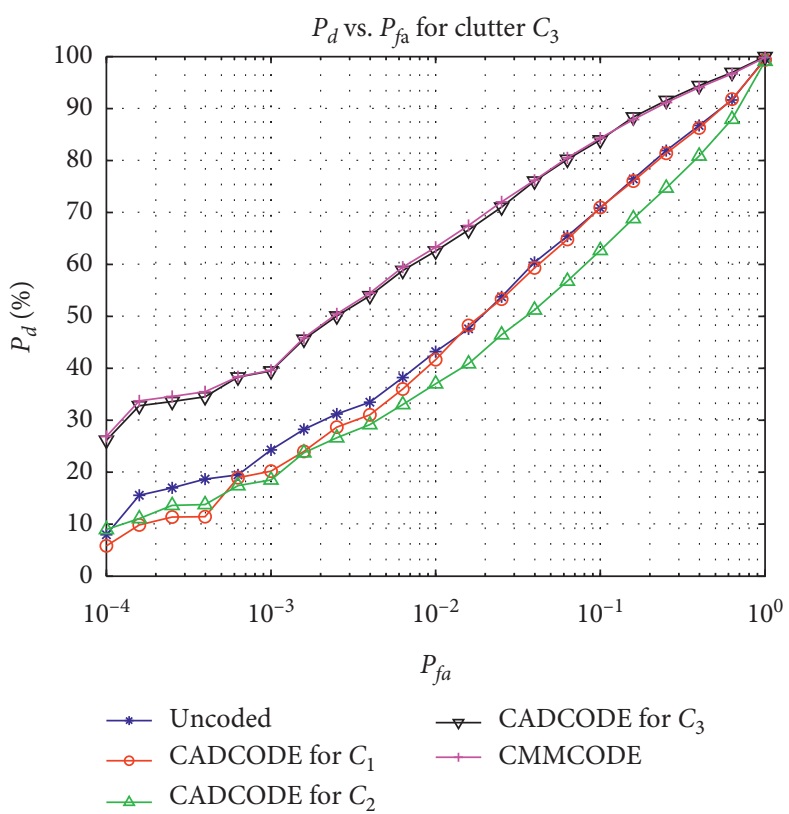

(c)

FIGURE 5: Probability of detection vs. probability of false alarm for 3 range cells with the resultant codes of CADCODE and CMMCODE algorithms and the uncoded system. (a) $P_{d}$ vs. $P_{f a}$ for clutter $C_{1}$; (b) $P_{d}$ vs. $P_{f a}$ for clutter $C_{2}$; and (c) $P_{d}$ vs. $P_{f a}$ for clutter $C_{3}$.

improvement is obtained when the transmit energy $e$ lies in $[4,10] \mathrm{dB}$.

Figures 5(a)-5(c) plot the receiver operating characteristic (ROC) curves, which is the probability of detection of the receiver as a function of the probability of false alarm, for 3 range cells with the resultant codes of CADCODE and CMMCODE algorithms, and the uncoded systems when transmit energy e equals to $5 \mathrm{~dB}$. It can be observed that the probability of detection of the resultant code of CMMCODE algorithm achieves good performance in 3 range cells, and the probability of detection of the resultant code of CADCODE algorithm can achieve a good performance in only one of the three range cells.

5.3. White-Noise Case. In this example, the signal-independent interference is assumed to be receiver noise, which is white and statistically independent with each other. The noise power $\sigma_{n}^{2}$ is 0.01 .

Figures 6(a)-6(b) show the average metric and detection performance of the resultant codes of the CADCODE and CMMCODE algorithms. We also note that the resultant codes in CMMCODE algorithm own the highest metric and 


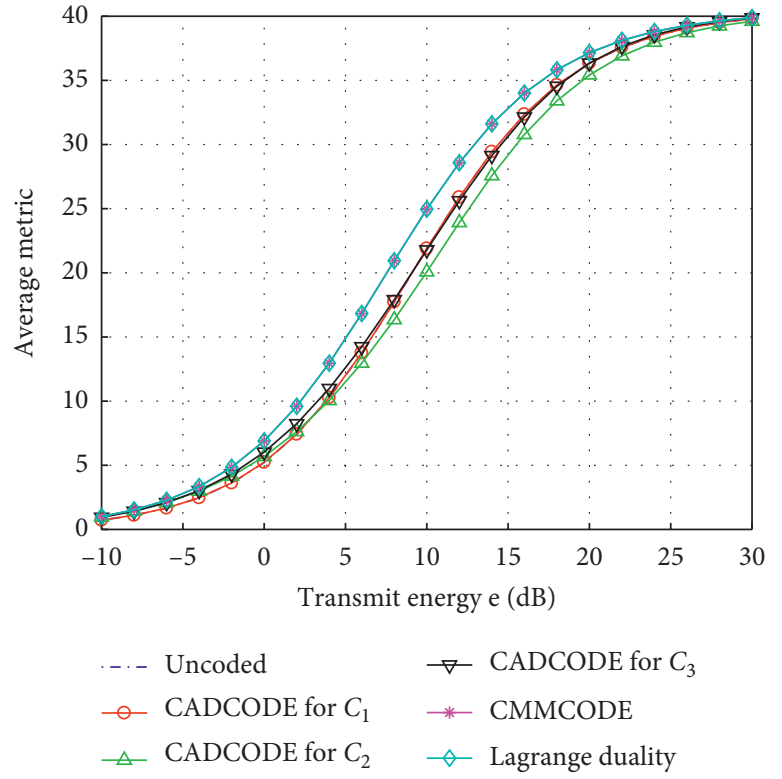

(a)

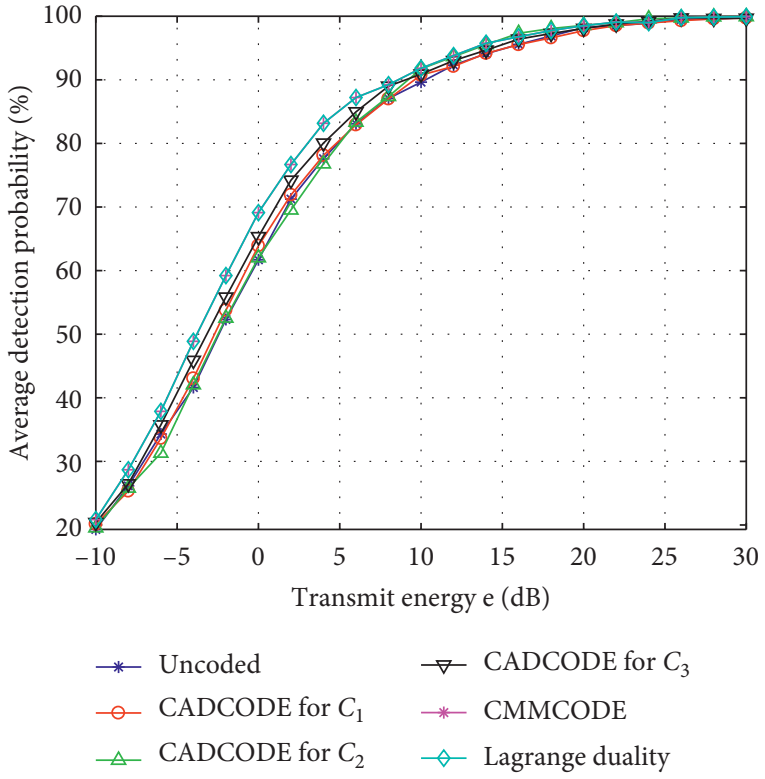

(b)

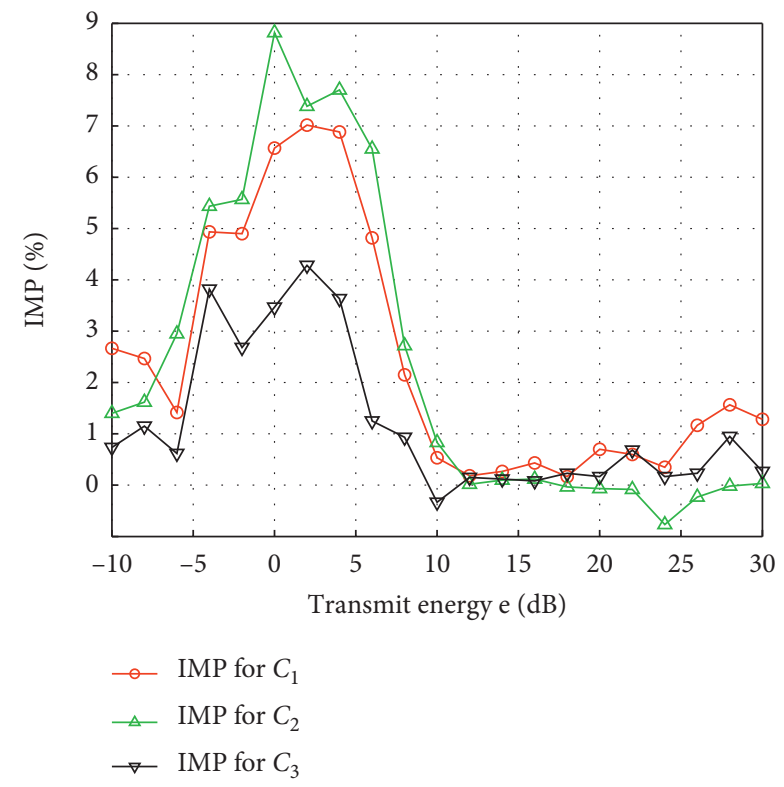

(c)

Figure 6: (a) Average metric of the resultant codes of CoRe, CADCODE, and CMMCODE algorithms and the uncoded system for 3 range cells; (b) average detection probability of an adaptive matched filter detector; and (c) IMP factor for different range cells.

best detection performance. The average metric and detection performance of the resultant codes in CADCODE algorithm is slightly lower than the former. In this simulation, the global solution of (29) obtained by the Lagrange duality method is compared with CMMCODE. As we can see, their performance is very close. Figure 6(c) demonstrates the metric performance of the resultant codes of CMMCODE algorithm, compared with that of the CADCODE algorithm. It can be observed that the average detection probability of the resultant code of CMMCODE algorithm is 9 percent higher than that of the resultant codes of CADCODE algorithm in the maximum extent. This improvement is not available in all transmit energy ranges, and the best improvement is obtained when the transmit energy $e$ equals to $0 \mathrm{~dB}$.

Figures $7(\mathrm{a})-7(\mathrm{c})$ plot the ROC curves for 3 range cells with the resultant codes of CADCODE and CMMCODE algorithms and the uncoded systems when the transmit energy $e$ equals to $0 \mathrm{~dB}$. It can also be observed that the probability of detection of the resultant code of CMMCODE algorithm achieves good performance in 3 range cells, and the probability of detection of the resultant code of CADCODE algorithm can achieve a good performance only in one of the three range cells. 


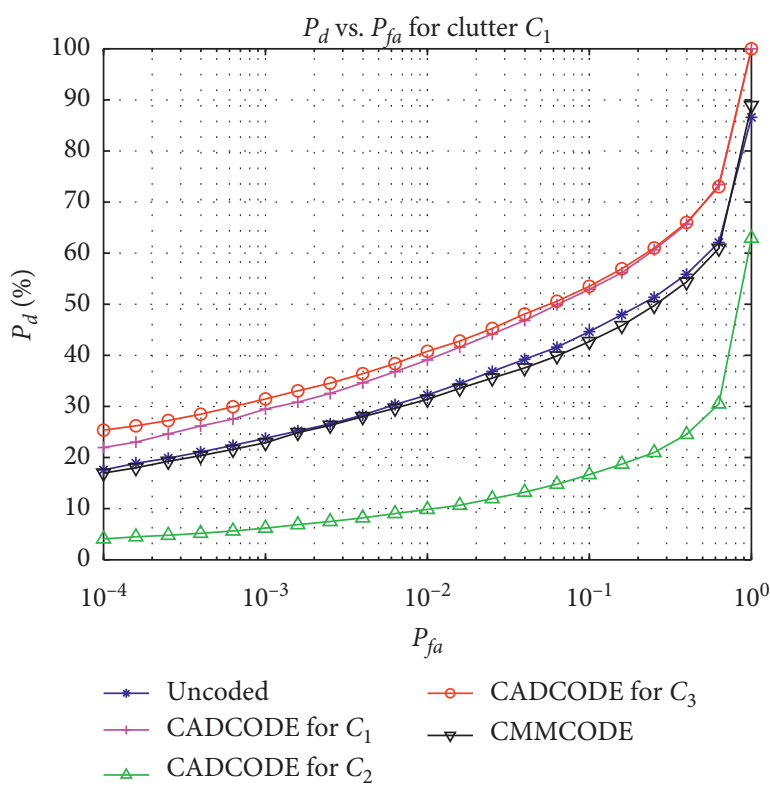

(a)

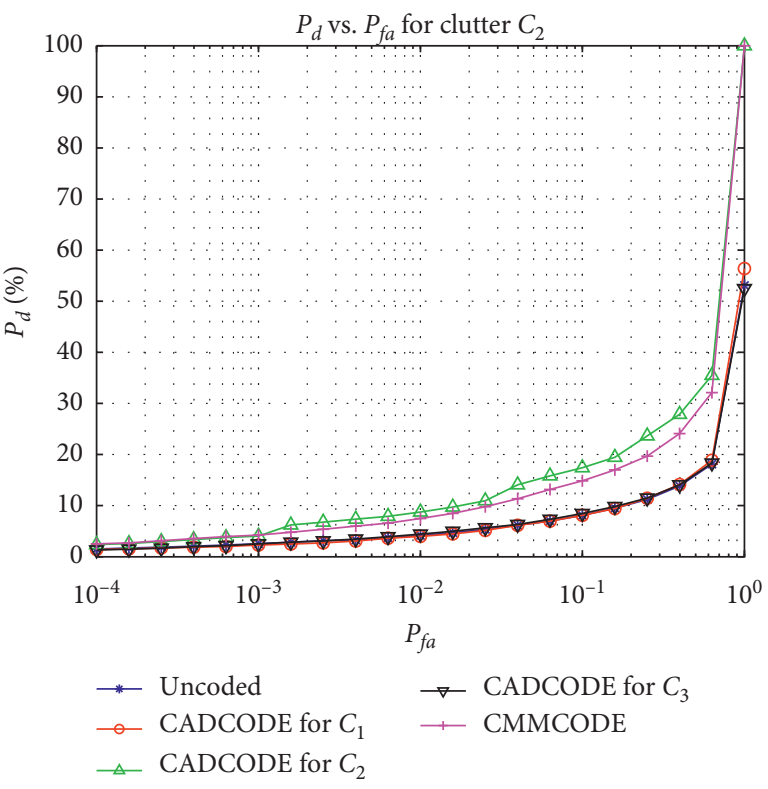

(b)

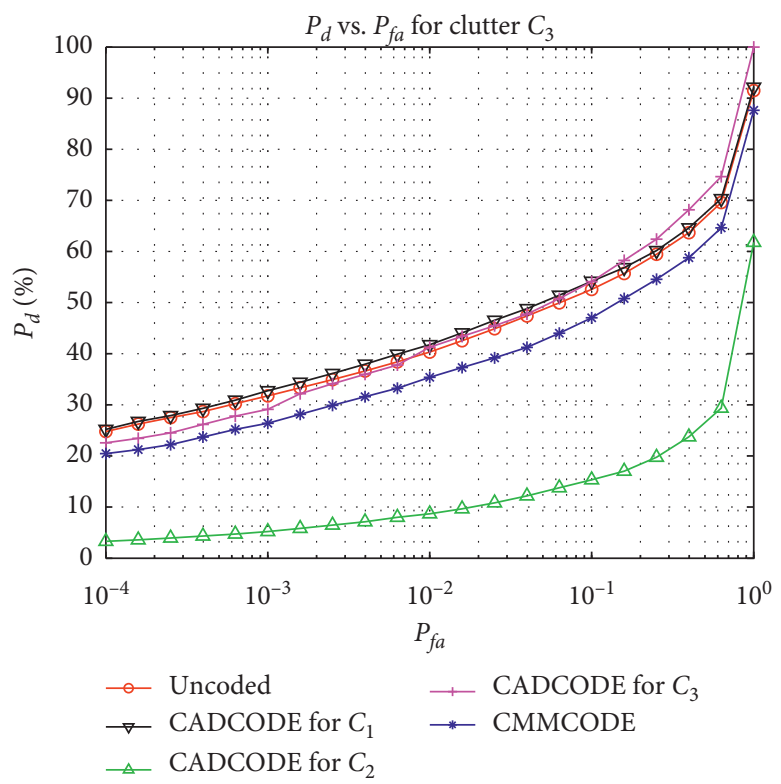

(c)

Figure 7: Probability of detection vs. probability of false alarm for 3 range cells with the resultant codes of CADCODE and CMMCODE algorithms and the uncoded system. (a) $P_{d}$ vs. $P_{f a}$ for clutter $C_{1}$; (b) $P_{d}$ vs. $P_{f a}$ for clutter $C_{2}$; and (c) $P_{d}$ vs. $P_{f a}$ for clutter $C_{3}$.

\section{Conclusions}

In this paper, code design problem for a MTD radar system in a nonhomogeneous clutter scenario is investigated. Considering the SCNR metric of an interpulse matched filtering output, we construct the corresponding optimization problem for optimizing a radar waveform in a CPI and transform the optimization problem by majorization-minimization. For solving this optimization problem, we propose an algorithm, i.e., CMMCODE, based on cyclic and majorization-minimization algorithms, which is computationally efficient. In the white-noise case, the simplified algorithm is also given based on CMMCODE algorithm.
CMMCODE algorithm demonstrates computational efficiency and fast convergence. In the homogeneous clutter case, its performance is as good as CoRe and CADCODE algorithms. In the nonhomogeneous clutter case, it has better balanced performances for different range cells.

\section{Appendix}

\section{A. Proof of Lemma 1}

Because of the (semi) negative definiteness of $\mathbf{R}-\sigma_{1} \mathbf{I}$, we have 


$$
\left(\mathbf{x}-\mathbf{x}_{0}\right)^{H}\left(\mathbf{R}-\sigma_{1} \mathbf{I}\right)\left(\mathbf{x}-\mathbf{x}_{0}\right) \leq 0,
$$

which implies

$$
\mathbf{x}^{H} \mathbf{R} \mathbf{x} \leq \sigma_{1} \mathbf{x}^{H} \mathbf{I} \mathbf{x}+2 \operatorname{Re}\left(\mathbf{x}^{H}\left(\mathbf{R}-\sigma_{1} \mathbf{I}\right) \mathbf{x}_{0}\right)+\mathbf{x}_{0}^{H}\left(\sigma_{1} \mathbf{I}-\mathbf{R}\right) \mathbf{x}_{0} .
$$

\section{B. Proof of Theorem 1}

With Lemma 1, $\mathbf{x}^{H} \mathbf{X Q X} \mathbf{X}^{H} \mathbf{x}$ is majorized by

$$
\begin{aligned}
\mathbf{x}^{H} \mathbf{X} \mathbf{Q} \mathbf{X}^{H} \mathbf{x} \leq \xi_{1} \mathbf{x}^{H} \mathbf{I} \mathbf{x}+\mathbf{x}^{H}\left(\mathbf{X} \mathbf{Q} \mathbf{X}^{H}-\xi_{1} \mathbf{I}\right) \mathbf{x}_{0} \\
\quad+\mathbf{x}_{0}^{H}\left(\mathbf{X} \mathbf{Q} \mathbf{X}^{H}-\xi_{1} \mathbf{I}\right) \mathbf{x}+\mathbf{x}_{0}^{H}\left(\xi_{1} \mathbf{I}-\mathbf{X} \mathbf{Q} \mathbf{X}^{H}\right) \mathbf{x}_{0} \\
\leq \mathbf{x}^{H} \mathbf{X} \mathbf{Q} \mathbf{X}^{H} \mathbf{x}_{0}+\mathbf{x}_{0}^{H} \mathbf{X} \mathbf{Q} \mathbf{X}^{H} \mathbf{x}-\mathbf{x}_{0}^{H} \mathbf{X} \mathbf{Q} \mathbf{X}^{H} \mathbf{x}_{0} \\
\quad+2 \xi_{1} e-2 \xi_{1} \operatorname{Re}\left(\mathbf{x}^{H} \mathbf{x}_{0}\right) .
\end{aligned}
$$

With Lemma 2, we note that

$$
\begin{aligned}
\left|\mathbf{x}^{H} \mathbf{X} \mathbf{Q} \mathbf{X}^{H} \mathbf{x}_{0}\right| & \leq \sqrt{\left(\mathbf{x}^{H} \mathbf{X} \mathbf{Q} \mathbf{X}^{H} \mathbf{x}\right) \cdot\left(\mathbf{x}_{0}^{H} \mathbf{X} \mathbf{Q} \mathbf{X}^{H} \mathbf{x}_{0}\right)} \\
\left|\mathbf{x}_{0}^{H} \mathbf{X} \mathbf{Q} \mathbf{X}^{H} \mathbf{x}\right| & \leq \sqrt{\left(\mathbf{x}^{H} \mathbf{X} \mathbf{Q} \mathbf{X}^{H} \mathbf{x}\right) \cdot\left(\mathbf{x}_{0}^{H} \mathbf{X} \mathbf{Q} \mathbf{X}^{H} \mathbf{x}_{0}\right)}, \\
2 \operatorname{Re}\left(\mathbf{x}^{H} \mathbf{X} \mathbf{Q} \mathbf{X}^{H} \mathbf{x}_{0}\right) & \leq\left|\mathbf{x}^{H} \mathbf{X} \mathbf{Q} \mathbf{X}^{H} \mathbf{x}_{0}\right|+\left|\mathbf{x}_{0}^{H} \mathbf{X} \mathbf{Q} \mathbf{X}^{H} \mathbf{x}\right| \\
& \leq 2 \sqrt{\left(\mathbf{x}^{H} \mathbf{X} \mathbf{Q} \mathbf{X}^{H} \mathbf{x}\right) \cdot\left(\mathbf{x}_{0}^{H} \mathbf{X} \mathbf{Q} \mathbf{X}^{H} \mathbf{x}_{0}\right) .}
\end{aligned}
$$

Hence, we can easily obtain $\operatorname{Re}\left(\mathbf{x}^{H} \mathbf{X} \mathbf{Q X}{ }^{H} \mathbf{x}_{0}\right) \leq \mathbf{x}^{H} \mathbf{X} \mathbf{Q} \mathbf{X}^{H} \mathbf{x} \quad$ and $\operatorname{Re}\left(\mathbf{x}^{H} \mathbf{X} \mathbf{Q} \mathbf{X}^{H} \mathbf{x}_{0}\right) \leq \mathbf{x}_{0}^{H} \mathbf{X} \mathbf{Q} \mathbf{X}^{H} \mathbf{x}_{0}$, and therefore, we have

$$
\mathbf{x}^{H} \mathbf{X} \mathbf{Q} \mathbf{X}^{H} \mathbf{x} \leq \mathbf{x}_{0}^{H} \mathbf{X} \mathbf{Q} \mathbf{X}^{H} \mathbf{x}_{0}+2 \xi_{1} e-2 \xi_{1} \operatorname{Re}\left(\mathbf{x}^{H} \mathbf{x}_{0}\right) \text {. }
$$

Note that $\xi_{1}$ is also the largest eigenvalue of $\mathbf{X}_{0} \mathbf{Q}^{*} \mathbf{X}_{0}^{H}$; we apply Lemma 2 again to the abovementioned inequality and obtain

$$
\mathbf{x}^{H} \mathbf{X} \mathbf{Q} \mathbf{X}^{H} \mathbf{x} \leq 2 \operatorname{Re}\left[\mathbf{x}^{H} \mathbf{X}_{0} \mathbf{Q}^{*} \mathbf{X}_{0}^{H} \mathbf{x}_{0}\right]-4 \xi_{1} \operatorname{Re}\left(\mathbf{x}^{H} \mathbf{x}_{0}\right)+\kappa\left(\mathbf{x}_{0}\right) .
$$

\section{Proof of Theorem 2}

It is easy to show that

$$
\begin{aligned}
& \nabla \tilde{f}\left(\mathbf{a}^{(\infty)}\right)=\nabla \widetilde{\mu}\left(\mathbf{a}^{(\infty)}, \mathbf{a}^{(\infty)}\right)=3 \mathbf{M a} \mathbf{a}^{(\infty) *} \\
& \quad+4 \mathbf{A}^{(\infty)} \mathbf{C}^{*} \mathbf{A}^{(\infty) H} \mathbf{a}^{(\infty) *} .
\end{aligned}
$$

Due to the positive definiteness of $\mathbf{M}$ and $\mathbf{C}$, we can easily obtain $\nabla \widetilde{f}\left(\mathbf{a}^{(\infty)}\right)^{T} \mathbf{a}^{(\infty)} \geq 0$, which indicates that the limit point is also a stationary point.

\section{Data Availability}

The data used to support this study are available within the article.

\section{Conflicts of Interest}

The authors declare that they have no conflicts of interest.

\section{Acknowledgments}

This work was supported by Fundamental Research Funds for Central Universities of China under grant no. NS2019024.

\section{References}

[1] M. Skolnik, Radar Handbook, McGraw-Hill, NewYork, NY, USA, 3rd edition, 2008.

[2] M. M. Naghsh, M. Soltanalian, P. Stoica, and M. ModarresHashemi, "Radar code design for detection of moving targets," IEEE Transactions on Aerospace and Electronic Systems, vol. 50, no. 4, pp. 2762-2778, 2014.

[3] D. DeLong and E. M. Hofstetter, "On the design of optimum radar waveforms for clutter rejection," IEEE Transactions on Information Theory, vol. 13, no. 3, pp. 454-463, 1967.

[4] P. G. Grieve and J. R Guerci, "Optimum matched illumination-reception radar," U.S. Patent S517552, 1992.

[5] S. U. Pillai, H. S. OH, D. C. Youla, and J. R. Guerci, “Optimal transmit-receiver design in the presence of signal-dependent interference and channel noise," IEEE Transactions on Information Theory, vol. 46, no. 2, pp. 577-584, 2000.

[6] D. A. Garren, M. K. Osborn, A. C. Odom, J. S. Goldstein, S. U. Pillai, and J. R. Guerci, "Enhanced target detection and identification via optimised radar transmission pulse shape," IEE Proceedings-Radar, Sonar and Navigation, vol. 148, no. 3, pp. 130-138, 2001.

[7] T. Naghibi and F. Behnia, "MIMO radar waveform design in the presence of clutter," IEEE Transactions on Aerospace and Electronic Systems, vol. 47, no. 2, pp. 770-781, 2011.

[8] P. Setlur and M. Rangaswamy, "Signal dependent clutter waveform design for radar STAP," in Proceedings of the IEEE Radar Conference, pp. 1311-1316, Cincinnati, OH, USA, May 2015.

[9] P. Setlur and M. Rangaswamy, "Projected gradient waveform design for fully adaptive radar STAP," in Proceedings of the IEEE Radar Conference, pp. 1704-1709, Arlington, VA, USA, May 2015.

[10] P. Setlur and M. Rangaswamy, "Proximal constrained waveform design algorithms for cognitive radar STAP," in Proceedings of the 48th Asilomar Conference on Signals, Systems and Computers, November 2014.

[11] P. Setlur and M. Rangaswamy, "Waveform design for radar STAP in signal dependent interference," IEEE Transactions on Signal Processing, vol. 64, no. 1, pp. 19-24, 2016.

[12] P. Setlur and M. Rangaswamy, "Waveform design for radar STAP in signal dependent interference," IEEE Transactions on Signal Processing, vol. 64, no. 1, pp. 19-34, 2016.

[13] A. De Maio, S. De Nicola, Y. Huang, Z.-Q. Luo, and S. Zhang, "Design of phase codes for radar performance optimization with a similarity constraint," IEEE Transactions on Signal Processing, vol. 57, no. 2, pp. 30-40, 2009.

[14] A. Aubry, A. DeMaio, A. Farina, and M. Wicks, "Knowledgeaided (potentially cognitive) transmit signal and receive filter design in signal-dependent clutter," IEEE Transactions on Aerospace and Electronic Systems, vol. 49, no. 1, pp. 93-117, 2013.

[15] A. De Maio, A. Yongwei Huang, M. Piezzo, and M. Piezzo, "A Doppler robust max-min approach to radar code design," IEEE Transactions on Signal Processing, vol. 58, no. 9, pp. 4943-4947, 2010.

[16] A. De Maio, Y. Huang, M. Piezzo, S. Zhang, A. Farina, and A. Farina, "Design of optimized radar codes with a peak to 
average power ratio constraint," IEEE Transactions on Signal Processing, vol. 59, no. 6, pp. 2683-2697, 2011.

[17] A. Aubry, M. Piezzo, A. De Maio, A. Farina, and M. Wicks, "Cognitive design of the receive filter and transmitted phase code in reverberating environment," IET Radar, Sonar \& Navigation, vol. 6, no. 9, pp. 822-833, 2012.

[18] S. M. Karbasi, A. Aubry, A. De Maio, and M. H. Bastani, "Robust transmit code and receive filter design for extended targets in clutter," IEEE Transactions on Signal Processing, vol. 63, no. 8, pp. 1965-1976, 2015.

[19] A. Aubry, A. De Maio, and M. M. Naghsh, "Optimizing radar waveform and Doppler filter bank via generalized fractional programming," IEEE Journal of Selected Topics in Signal Processing, vol. 9, no. 8, pp. 1387-1399, 2015.

[20] X. Cheng, A. Aubry, D. Ciuonzo, A. De Maio, and X. Wang, "Robust waveform and filter bank design of polarimetric radar," IEEE Transactions on Aerospace and Electronic Systems, vol. 53, no. 1, pp. 370-384, 2017.

[21] M. Razaviyayn, M. Hong, and Z.-Q. Luo, "A unified convergence analysis of block successive minimization methods for nonsmooth optimization," SIAM Journal on Optimization, vol. 23, no. 2, pp. 1126-1153, 2013.

[22] A. Aubry, A. De Maio, A. Zappone, M. Razaviyayn, and L. Zhi-Quan, "A new sequential optimization procedure and its applications to resource allocation for wireless systems," IEEE Transactions on Signal Processing, vol. 66, no. 24, pp. 6518-6533, 2018.

[23] L. Luo and D. P. Palomar, "Maximin joint optimization of transmitting code and receiving filter in radar and communications," IEEE Transactions on Signal Processing, vol. 65, no. 4, pp. 850-863, 2016.

[24] L. Wu, P. Babu, and D. P. Palomar, "Transmit waveform/ receive filter design for MIMO radar with multiple waveform constraints," IEEE Transactions on Signal Processing, vol. 66, no. 6, pp. 1526-1540, 2018.

[25] J. Song, D. P. P. Babu, and D. P. Palomar, "Sequence design to minimize the weighted integrated and peak sidelobe levels," IEEE Transactions on Signal Processing, vol. 64, no. 8, pp. 2051-2064, 2016.

[26] M. M. Naghsh, M. Soltanalian, M. Modarres-Hashemi, P. Stoica, and A. Aubry, "A doppler robust design of transmit sequence and receive filter in the presence of signal-dependent interference," IEEE Transactions on Signal Processing, vol. 62, no. 4, pp. 772-785, 2014.

[27] X. De Maio, A. Aubry, A. De Maio, and G. Cui, "Hidden convexity in robust waveform and receive filter bank optimization under range unambiguous clutter," IEEE Signal Processing Letters, vol. 27, 2020.

[28] P. Stocia, H. He, and J. Li, "New algorithms for designing unimodular sequences with good correlation properties," IEEE Transactions on Signal Processing, vol. 57, no. 4, pp. 1415-1425, 2009.

[29] M. Soltanalian and P. Stoica, "Computational design of sequences with good correlation properties," IEEE Transactions on Signal Processing, vol. 60, no. 5, pp. 2180-2193, 2012.

[30] P. Stoica, H. Hao He, and J. Jian Li, "On designing sequences with impulse-like periodic correlation," IEEE Signal Processing Letters, vol. 16, no. 8, pp. 703-706, 2009.

[31] M. Soltanalian and P. Stoica, "Designing unimodular codes via quadratic optimization," IEEE Transactions on Signal Processing, vol. 62, no. 5, pp. 1221-1234, 2014.

[32] D. R. Hunter and K. Lange, "A tutorial on MM algorithms," The American Statistician, vol. 58, no. 1, pp. 30-37, 2004.
[33] P. Stoica and Y. Selen, "Cyclic minimizers, majorization techniques, and the expectation-maximization algorithm: a refresher," IEEE Signal Processing Magazine, vol. 21, no. 1, pp. 112-114, 2004.

[34] J. R. Guerci, Cognitive Radar, the Knowledge-Aided Fully Adaptive Approach, Artech House, Norwood, MA, USA, 2010.

[35] S. M. Kay, Fundamentals of Statistical Signal ProcessingVolume II: Detection Theory, Prentice-Hall, Upper Saddle River, NJ, USA, 1st edition, 1998.

[36] J. Michael Steele, The Cauchy-Schwarz Master Class: An Introduction to the Art of Mathematical Inequalities, Cambridge University Press, Cambridge, UK, 2004.

[37] T. Foregger, A. Ismail, and P. Sanchez, "MacLaurin's inequality (version 4)," PlanetMath. org, 2018, http:// planetmath.org/MacLaurinsInequality.html.

[38] T. J. Nohara and S. Haykin, "Growler detection in sea clutter using Gaussian spectrum models," IEE Proceedings-Radar, Sonar and Navigation, vol. 141, no. 5, pp. 285-292, 1994.

[39] F. C. Robey, D. R. Fuhrmann, E. J. Kelly, and R. Nitzberg, "A CFAR adaptive matched filter detector," IEEE Transactions on Aerospace and Electronic Systems, vol. 28, no. 1, pp. 208-216, 1992.

[40] E. Conte, M. Lops, and G. Ricci, "Adaptive matched filter detection in spherically invariant noise," IEEE Signal Processing Letters, vol. 3, no. 8, pp. 248-250, 1996. 